\title{
Evolution im Operationssaal
}

\section{Evolution in the Operating Room}

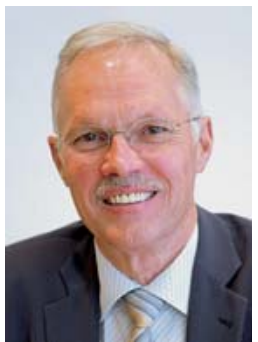

F. U. Niethard

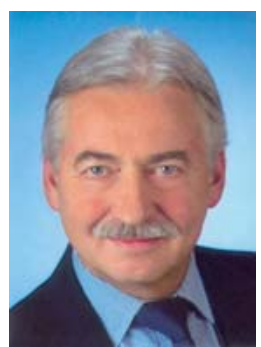

K. Weise

\section{Bibliografie}

DOI 10.1055/s-0029-1240679 Z Orthop Unfall 2009; 147: 667668 @ Georg Thieme Verlag KG Stuttgart · New York . ISSN 1864-6697

\section{Korrespondenzadressen}

Prof. Dr. med. Fritz U. Niethard Orthopädische Klinik

Universitätsklinik der RWTH Aachen

Pauwelsstraße 30

52074 Aachen

Tel.: 0241/808-94 10

Fax: 0241/808-2453

funiethard@

orthopaedie-aachen.de

Prof. Dr. med. Kuno Weise BG-Unfallklinik

Schnarrenbergstraße 95

72076 Tübingen

Tel.: 07071/606-1001

Fax: 07071/606-1002

weise@bgu-tuebingen.de
Noch nie hat ein technologischer Fortschritt vor der Medizin haltgemacht, so auch nicht der Computer. Computer haben die ärztliche Praxis und das Krankenhaus längst erobert. Und dennoch waren die ersten Roboter im Operationssaal eine „Revolution“. Zum ersten Mal ist der Computer direkt in das Arzt-Patienten-Verhältnis eingedrungen. Der Operationsroboter hat sich angemaßt, präziser und damit sogar besser zu sein als die menschliche Hand. Diese Zeitschrift hat sich in zahlreichen Artikeln mit dieser Thematik beschäftigt. In Editorials wurde davor gewarnt, dass die primär euphorisch begrüßte Technologie ausschließlich zu Marketingzwecken missbraucht werde und damit im Chaos enden könnte [3]. Zu einer analog der in den USA von der Food and Drug Administration ausgesprochenen beschränkten Zulassung der Technologie kam es nicht, sodass vor allem falsche Indikationsstellungen und ungeeignete Operationsstrategien zum Niedergang der Robotertechnologie geführt haben.

Demgegenüber hat sich die computergestützte Navigation beim Hüft- und Kniegelenkersatz stetig weiterentwickelt, denn schonende Operation heißt die Herausforderung an das Chirurgenteam von morgen. Der kleine Schnitt ist sinnbildlich geworden für den schonenden Eingriff, die rasche Genesung des Patienten und damit auch für ein kosteneffizientes Gesundheitssystem. Wenn der Operateur aber bei minimalinvasiven Zugängen eine eingeschränkte Sicht auf das Operationsumfeld hat, dann werden Navigationshilfen, neue Bilddarstellungsformen und technisch intelligente Instrumente benötigt. Auch hierzu sind in der Zeitschrift für Orthopädie bzw. Zeitschrift für Orthopädie und Unfallchirurgie zahlreiche Artikel erschienen. Ihr gemeinsamer Tenor war, dass sich mit Navigationshilfen die Präzision des operativen Eingriffs verbessern lässt, ein genereller Einsatz vor allen Dingen an Kostenfaktoren scheiterte.

Diesen beiden Gesichtspunkten wenden sich nun die Beiträge von Prymka und Hassenflug [5] sowie von König et al. [2] zu. Prymka und Hassenflug berichten in ihrer Studie erstmals über längerfristige, nämlich 6-Jahres-Ergebnisse nach roboterunterstützter Hüftendoprothesenimplantation. Die Ergebnisse bei 32 Patienten werden von ihnen sehr positiv bewertet. Vor allem die allseits mit der Roboterimplantation in Verbindung gebrachten glutealen Insuffizienzen traten nicht auf. Auch Schmerzbefunde im Bereich der zur Referenzierung eingebrachten vormaligen Schraubenkanäle wurden nicht beobachtet. Damit reihen sie sich in die Gruppe derjenigen Autoren ein, die die Probleme nach der Implantation vor allem auf Anwenderfehler zurückführen [1].

König und Mitarbeiter haben sich in ihrer Arbeit mit einer breiten Anwendung der Navigation entgegenstehenden Kostenfrage beschäftigt. Sie konnten belegen, dass wie in zahlreichen anderen Publikationen auch die navigierten Knie- und Hüftendoprothesen präziser implantiert werden konnten. Diesem für den Patienten bedeutsamen Nutzen steht ein vermeintlich höherer Kostenaufwand gegenüber, da die Operationsdauer signifikant verlängert ist. Allerdings steht zu vermuten, dass eine optimale Positionierung der Implantate auch mit einer verlängerten Überlebenszeit, mit niedrigeren Revisionsraten und damit auch niedrigeren Krankenfolge- und Arbeitsunfähigkeitskosten einhergeht. Volkswirtschaftlich betrachtet wäre somit auch die Navigation kostensparend. Eine Bestätigung durch derartige notwendigerweise längerfristig laufende Untersuchungen steht allerdings noch aus.

Wie wird der orthopädisch-/unfallchirurgische Operationssaal der Zukunft aussehen [4]? Prymka und Hassenflug bedauern, dass nicht schon bei den verbreiteten Robotersystemen eine saubere prospektive Dokumentation und höhere Transparenz der Behandlungen einschließlich einer vollständig dokumentierten Erfassung auch von Negativergebnissen stattgefunden hat. König und Mitarbeiter fordern Langzeitstudien, die die erwartete längere Prothesenstandzeit bei navigierten Eingriffen und somit eine geringere Revisionsrate als volkswirtschaftlichen Nutzen belegen könnten. Ziel ist also nicht etwa die von Marketinggesichtspunkten gelenkte „Revolution“, sondern die von öffentlichen Institutionen und Fachgesellschaften begleitete „Evolution im Operationssaal“.

\section{F. U. Niethard, Aachen}

K. Weise, Tübingen 


\section{Literatur}

1 Bach CM, Winter P, Nogler $M$ et al. No functional impairment after Robodoc total hip arthroplasty: gait analysis in 25 patients. Acta Orthop Scand 2002; 73: 386-391

2 König DP, Michael J, Eysel P et al. Navigation in der Endoprothetik. Die Kosten-Nutzen-Analyse einer orthopädischen Fachklinik. Z Orthop Unfall 2009; 147: 669-674
3 Niethard FU. Der „neue Markt“ und die computerassistierte orthopädische Chirurgie - endet die CAOS im Chaos? Z Orthop 2001; 139: 95-96 4 Niethard FU. Navigation und Robotik. Implant 2008; 1: 2

5 Prymka M, Hassenpflug J. 6-Jahres-Ergebnisse nach roboterunterstützter Hüftendoprothesenimplantation - eine prospektive Studie. Z Orthop Unfall 2009; 147: 675-682 